Supporting Information:

\title{
Controllable Display of Sequential Enzymes on Yeast Surface with Enhanced Biocatalytic Activity towards Efficient Enzymatic Biofuel Cells
}

Shuqin Fan ${ }^{\dagger, \Sigma, \|}$, Bo Liang $^{\Sigma, \|}$, Xinxin Xiao ${ }^{\dagger, \|}$, Lu Bai $^{\dagger}$, Xiangjiang Tang ${ }^{\Sigma}$, Elisabeth Lojou $^{\Delta, *}$, Serge Cosnier ${ }^{\zeta} \eta, *$, and Aihua Liu ${ }^{\dagger, \star}, *$

$\dagger$ Institute for Chemical Biology \& Biosensing, and College of Life Sciences, Qingdao University, 308 Ningxia Road, Qingdao 266071, China

${ }^{\ddagger}$ School of Pharmacy, College of Medicine, 308 Ningxia Road, Qingdao 266071, China

${ }^{\Sigma}$ Qingdao Institute of Bioenergy \& Bioprocess Technology, Chinese Academy of Sciences, 189 Songling Road, Qingdao 266101, China

${ }^{\Delta}$ Aix Marseille Univ, CNRS, BIP, Bioénergétique et Ingénierie des Protéines UMR7281, 31 chemin Joseph Aiguier 13402 Marseille Cedex 20, France

${ }^{\zeta}$ Université Grenoble-Alpes, DCM UMR 5250, F-38000 Grenoble, France

${ }^{\eta}$ Département de Chimie Moléculaire, UMR CNRS, DCM UMR 5250, F-38000

Grenoble, France

"These authors contributed equally to this work.

\section{Corresponding Authors}

*Elisabeth Lojou, E-mail: lojou@imm.cnrs.fr;

*Serge Cosnier, E-mail: serge.cosnier@univ-grenoble-alpes.fr;

*Aihua Liu, E-mail: liuah@qdu.edu.cn.

Table of Contents:

1. Supplementary Experimental Methods

2. Supplementary Tables

3. Supplementary Figures 


\section{Supplementary Experimental Methods}

1.1 Strains, media and chemicals

The recombinant strains and plasmids in the present study were listed in Table S1. Escherichia coli DH5 $\alpha$ (F- $\varphi 80$ lacZ $\Delta$ M15 (lacZYA-argF) U169 endA1 recA1 hsdR17 (rk-,mk+)supE44 $\lambda$-thi-1 gyrA96 relA1 phoA) was used for recombinant DNA manipulation. S. cerevisiae EBY100 and Pichia pastoris X-33 (Invitrogen, Carlsbad, CA) were used for yeast cell surface display of the scaffoldins and secreted expression of recombinant proteins. E. coli was cultured in Luria-Bertani (LB) medium supplemented with ampicillin (100 $\mu \mathrm{g}$ $\left.\mathrm{mL}^{-1}\right)$ or zeocin $\left(25 \mu \mathrm{g} \mathrm{mL}^{-1}\right)$. Yeasts were grown in YPD media. S. cerevisiae EBY100 transformants were selected on SD-trp plates, and galactose was used to replace glucose in SD-trp media for protein expression induction. P. pastoris X-33 transformants were selected on YPDS plates supplemented with zeocin at $100 \mu \mathrm{g} \mathrm{mL}-1$. The recombinant cells were percultured in BMGY medium, and then induced in BMMY with $1 \%$ methanol and $10 \mathrm{mM}$ $\mathrm{CaCl}_{2}$. All restriction enzymes were obtained from Fermentas (MBI Fermentas, Canada). Graphene was kindly given by Dr. Liwei Liu in Suzhou Institute of Nano-tech and Nanobionics, Chinese Academy of Sciences. Poly(acrylic acid) (PAA) and 2, 2'-azinobis (3ethylbenzothiazoline-6-sulfonic acid) diammonium salt (ABTS) were obtained from Aladdin Reagent Company (Shanghai, China). D-glucose, starch and methylene blue (MB) were purchased from Sinopharm Chemical Reagent Co., Ltd (Shanghai, China). Cation exchange membrane was purchased from the Green Environmental Protection Technology Co., Ltd (Hangzhou, China). Laccase (Lac) from Trametes versicolor and Nafion ${ }^{\circledR} 117$ solution containing 5\% Nafion were purchased from Sigma-Aldrich (St. Louis, USA). Lac was purified using a dialysis membrane (cutoff molecular weight: $10 \mathrm{kD}$ ) before use. The specific activity of Lac is 13.6 and $21.2 \mathrm{U} \mathrm{mg}^{-1}$ for before and after dialysis, respectively. All other chemicals were used as received without further purification. Ultrapure water was prepared using a Milli-Q system (Millipore, Billerica, MA, USA).

1.2 Construction, assay and evaluation of the displayed sequential enzyme systems

\subsubsection{Plasmid construction}

Primers used for plasmid construction are summarized in Table S2. Plasmid pYD1 and $\mathrm{pPICZ} \alpha \mathrm{A}$ were used as the parent vector for construction and expression of fusion protein. Plasmids bearing the genes of Coh-Doc pair proteins were presented by Dr. Yingang Feng in QIBEBT, Qingdao. A gene encoding scaffoldin with CohC-CohT was obtained by performing PCR using primer pair cohC-cohT For/Rev using pET28NS-C1-T3 as the template. The 
amplified fragment was cloned into Nhe I/Bam HI-digested plasmid pYD1 to form pYD1cohC-cohT. CohC encoding gene was amplified using primer pair cohC-F1 For/ cohC-R1 Rev. The product was digested by Xho I and Apa I and ligated into pYD1-cohC- cohT to form pYD1-cohC-cohT-cohC. To obtain plasmid pYD1-cohC-cohC-cohT, the fragment of cohC gene was amplified by PCR with primers cohC-F2 For and cohC-R2 Rev, which was cut with Nhe I and Bam HI and ligated into the corresponding sites of pYD1, and then the gene fragment of cohC-cohT was amplified with primers cohC-cohT2 For/Rev, which was cut with Bam HI and Xho I and ligated into the corresponding sites of above vector. In order to construct plasmid pYD1-cohC-cohC-cohT-cohC, CohC encoding gene was amplified with primers cohC-F1 For and cohC-R1 Rev, which was cut with Xho I and Apa I and ligated into the corresponding sites of pYD1-cohC-cohC-cohT. In order to construct plasmid pYD1-cohCcohC-cohT-cohC, the fragment of cohc gene was amplified by PCR with primers cohC-F2 For and cohC-R2 Rev, which was cut with Nhe I and Bam HI and ligated into the corresponding sites of pYD1-cohC-cohT-cohC. These plasmids were transformed in S. cerevisiae EBY100 using the standard lithium acetate procedure. Corresponding DocC and DocT encoding genes were amplified by PCR using docC For/Rev, docT For/Rev as primers from pET28NS-docC and pET28NS-docT, respectively. S. fibuligera genomic DNA was obtained from Prof. Zhenming Chi (Ocean University of China, Qingdao) as a gift. Gene fragment coding for GA were amplified with S. fibuligera genomic DNA as the template using primer pair ga For/ Rev, and GOx encoding gene was amplified by PCR from genomic DNA of A. niger using primers gox For and gox Rev. GA gene and DocC gene were fused by Overlap Extension PCR to obtain GA-DocC fusion gene, which was inserted into pPICZ $\alpha A$ vector to form pPICZ $\alpha \mathrm{A}$-ga-docC. To generate pPICZ $\alpha \mathrm{A}$-gox-docT, the same method was used. Both of two secreted expression vectors were separately transformed in P. pastoris X-33 by electroporation method.

\subsubsection{Enzyme assays}

starch $\stackrel{\text { GA }}{\rightarrow}$ glucose

glucose $+\mathrm{O}_{2} \stackrel{\text { GOx }}{\longrightarrow}$ gluconolactone $+\mathrm{H}_{2} \mathrm{O}_{2}$

GA activity was measured at room temperature for $15 \mathrm{~min}$ in $50 \mathrm{mM} \mathrm{HAc}-\mathrm{NaAc}$ buffer (pH 5.0) containing $0.1 \%$ soluble starch. Glucose was detected using glucose detection kit bought from Jiancheng, China. GA enzyme activity (one unit) is defined as the enzyme amount that generates $1 \mu \mathrm{mol}$ glucose per min under the given assay conditions. GOx activity assay was used as described before [1]. Briefly, enzyme samples were added into $100 \mathrm{mM}$ phosphate buffer ( $\mathrm{pH}$ 7.4) containing $10 \mathrm{mM}$ glucose, $0.17 \mathrm{mM} o$-dianisidine dihydrochloride and $2 \mathrm{U}$ 
$\mathrm{mL}^{-1}$ horseradish peroxidase (HRP). The enzymatic reaction was carried out at room temperature. Then the absorbance at $500 \mathrm{~nm}$ was recorded. One unit of GOx activity is defined as the enzyme amount that $1 \mu \mathrm{mol} \mathrm{H}_{2} \mathrm{O}_{2}$ per minute under the above assay condition.

\subsubsection{The overall reaction rate of GA\&GOx-yeast catalyzing starch}

Starch was used as substrate, which could be finally transformed into D-gluconolactone and hydrogen peroxide by GA\&GOx-yeast (eqs.1, 2). The overall reaction was conducted for 15 min in $50 \mathrm{mM}$ HAc-NaAc buffer $(1 \mathrm{~mL}, \mathrm{pH} 5.0)$ containing reaction cocktail $(0.1 \%$ soluble starch, $0.17 \mathrm{mM} o$-dianisidine dihydrochloride, $2 \mathrm{U} \mathrm{mL}^{-1} \mathrm{HRP}$ ) and GA\&GOx-yeast cells $\left(\mathrm{OD}_{600 \mathrm{~nm}}=1\right)$. The overall reaction rate $\left(\mathrm{nmol} \mathrm{H}_{2} \mathrm{O}_{2} \mathrm{~min}^{-1} \mathrm{~mL}^{-1}\right)$ is defined as the amount of generated $\mathrm{H}_{2} \mathrm{O}_{2}\left(\mathrm{nmol} \mathrm{mL} \mathrm{m}^{-1}\right)$ under the above assay condition. All the experiments were repeated at least three times.

\subsubsection{Estimation of expressed GA and GOx numbers on the yeast cell surface}

The calculation of the apparent numbers of enzymes displayed per cell was carried out based on the the previous reports $[2,3]$. The activity of the immobilized enzymes on the cell surfaces is assumed to be the same as that of the free enzymes. To determine the copy number of GA and GOx enzymes on the yeast cells surface, enzymatic activity assays were conducted using various amounts of purified dockerin-fused GA and dockerin-fused-GOx, respectively. The calibration curves were obtained by plotting the enzyme activities varying with the amounts of purified dockerin-fused enzymes ranging from 0 to $150 \mu \mathrm{g}$. The amounts of expressed enzymes on the surface of the strains were estimated on basis of the calibration curves. Finally, the numbers of displayed enzyme molecules on the cell surface were calculated on the basis of the molecular weights of dockerin-GA and dockerin-GOx along with the numbers of yeast cells.

\subsubsection{Testing the stability of engineered yeast}

Yeast cells co-displayed GA and GOx were incubated at $4{ }^{\circ} \mathrm{C}$ and room temperature, respectively. To evaluate the activity at $4^{\circ} \mathrm{C}$, it is necessary to bring the sample back to the room temperature, to measure and then to return the samples to $4{ }^{\circ} \mathrm{C}$. Meanwhile, the mixture of the same amount of free enzymes was treated under the same conditions. Over a period of 30 days, equal volume of each sample solution was taken to check the overall reaction rate as described above every 5 days.

1.3 Construction of EBFCs and their performance testing

\subsubsection{Preparation of PAA dispersed graphene}


PAA-graphene complex was prepared by dispersing $2 \mathrm{mg}$ graphene into $1 \mathrm{mg} \mathrm{mL}^{-1} \mathrm{PAA}$ in water under vigorous sonication for $30 \mathrm{~min}$ until a homogeneous dispersion resulted.

\subsubsection{Apparatus and electrochemical measurements}

Electrochemical measurements were carried out with a CHI660E potentiostat $(\mathrm{CH}$ Instruments, Chenhua, Shanghai, China). The half-cell measurement was performed in a conventional three-electrode system using the as-prepared electrode as working electrode, a saturated calomel electrode (SCE) as reference electrode, and a Pt wire electrode as auxiliary electrode. All potentials were reported in this paper were recorded versus this reference. 


\section{Supplementary Tables:}

Table S1. Strains and plasmids used in this study

\begin{tabular}{|c|c|c|}
\hline Strain & Plasmid & Description \\
\hline EBY & pYD1 & No surface display (negative control) \\
\hline $\mathrm{EBY}-\mathrm{C}_{1} \mathrm{~T}_{1}$ & pYD1-cohC-cohT & $\begin{array}{l}\text { Surface display of scaffoldin with CohC-CohT } \\
\text { on S. cerevisiae EBY } 100\end{array}$ \\
\hline EBY- $\mathrm{C}_{1} \mathrm{~T}_{1} \mathrm{C}_{1}$ & pYD1-cohC-cohT-cohC & $\begin{array}{l}\text { Surface display of scaffoldin with CohC-CohT- } \\
\text { CohC on S. cerevisiae EBY100 }\end{array}$ \\
\hline $\mathrm{EBY}-\mathrm{C}_{2} \mathrm{~T}_{1}$ & pYD1-cohC-cohC-cohT & $\begin{array}{l}\text { Surface display of scaffoldin with CohC-CohC- } \\
\text { CohT on S. cerevisiae EBY } 100\end{array}$ \\
\hline EBY- $\mathrm{C}_{2} \mathrm{~T}_{1} \mathrm{C}_{1}$ & pYD1-cohC-cohC-cohT-cohC & $\begin{array}{l}\text { Surface display of scaffoldin with CohC-CohC- } \\
\text { CohT-CohC on S. cerevisiae EBY } 100\end{array}$ \\
\hline X33-GA & pPICZ $\alpha A$-ga-docC & $\begin{array}{l}\text { Secreted expression of GA-DocC fusion protein } \\
\text { in P. pastoris }\end{array}$ \\
\hline X33- GOx & pPICZ $\alpha A$-gox-docT & $\begin{array}{l}\text { Secreted expression of GOx-DocT fusion protein } \\
\text { in P. pastoris }\end{array}$ \\
\hline
\end{tabular}


Table S2. PCR primers used in this study

\begin{tabular}{|c|c|}
\hline Primer & Sequence $\left(5^{\prime} \rightarrow 3^{\prime}\right)$ \\
\hline $\operatorname{coh} C$ - $\operatorname{coh} T$ For & CTAGCTAGCATCCCTGGCGATTCTCTTAAAG \\
\hline \multirow{2}{*}{$\operatorname{coh} C-\operatorname{coh} T \operatorname{Rev}$} & CGGGATCCTCACAGATCCTCTTCTGAGATGAGTTTTTGTTCAGGTGTTGT \\
\hline & AGGTGTTGTAGG \\
\hline $\operatorname{cohC}$-F1 For & CCGCTCGAGATCCCTGGCGATTCTCTTAAAG \\
\hline $\operatorname{coh} C$-R1 Rev & TCCGGGCCCTTGAGTACCAGGATCTATAGTTACAC \\
\hline $\operatorname{cohC}$-F2 For & CTAGCTAGCATCCCTGGCGATTCTCTTAAAG \\
\hline cohC-R2 Rev & CGGGATCCTTGAGTACCAGGATCTATAGTTACAC \\
\hline$g a$ For & GGAATTCATGAACACTGGACATTTCC \\
\hline$g a \operatorname{Rev}$ & CAATTACTGGGTCAGGATCTGTAAGAAGTTCAATCAATTT \\
\hline gox For & CCGCTCGAGAAAAGAGAGGCTGAAGCTAGGAGCAATGGCATTGAAG \\
\hline gox $\operatorname{Rev}$ & GACGTCGCCGTATAATTTAGTCTGCATGGAAGCATAATCTTCCAAG \\
\hline docC For & AAATTGATTGAACTTCTTACAGATCCTGACCCAGTAATTG \\
\hline $\operatorname{doc} C \operatorname{Rev}$ & GGGGTACCGTGTTGCTTGGAAGCTTACTTACC \\
\hline docT For & CTTGGAAGATTATGCTTCCATGCAGACTAAATTATACGGCGACGTC \\
\hline doct Rev & AAGGAAAAAAGCGGCCGCGTTCTTGTACGGCAATGTATC \\
\hline
\end{tabular}


Table S3. Amino acid sequences of dockerin-fused proteins

\begin{tabular}{|c|c|}
\hline dockerin-fused protein & Amino acid sequence \\
\hline \multirow[t]{13}{*}{ GA-DocC } & MNTGHFQAYSGYTVARSNFTQWIHEQPAVSWYYLLQNIDYPEG \\
\hline & QFKSAKPGVVVASPSTSEPDYFYQWTRDTAITFLSLIAEVEDHSF \\
\hline & SNTTLAKVVEYYISNTYTLQRVSNPSGNFDSPNHDGLGEPKFNV \\
\hline & DDTAYTASWGRPQNDGPALRAYAISRYLNAVAKHNNGKLLLAG \\
\hline & QNGIPYSSASDIYWKIIKPDLQHVSTHWSTSGFDLWEENQGTHF \\
\hline & FTALVQLKALSYGIPLSKTYNDPGFTSWLEKQKDALNSYINSSG \\
\hline & FVNSGKKHIVESPQLSSRGGLDSATYIAALITHDIGDDDTYTPFN \\
\hline & VDNSYVLNSLYYLLVDNKNRYKINGNYKAGAAVGRYPEDVYN \\
\hline & GVGTSEGNPWQLATAYAGQTFYTLAYNSLKNKKNLVIEKLNYD \\
\hline & LYNSFIADLSKIDSSYASKDSLTLTYGSDNYKNVIKSLLQFGDSF \\
\hline & LKVLLDHIDDNGQLTEEINRYTGFQAGAVSLTWSSGSLLSANRA \\
\hline & RNKLIELLTDPDPVIVYGDYNNDGNVDALDFAGLKKYIMAADH \\
\hline & AYVKNLDVNLDNEVNAFDLAILKKYLLGMVSKLPSN \\
\hline \multirow[t]{16}{*}{ GOx-DocT } & MRSNGIEASLLTDPKDVSGRTVDYIIAGGGLTGLTTAARLTENPN \\
\hline & ISVLVIESGSYESDRGPIIEDLNAYGDIFGSSVDHAYETVELATNN \\
\hline & QTALIRSGNGLGGSTLVNGGTWTRPHKAQVDSWETVFGNEGW \\
\hline & NWDNVAAYSLQAERARAPNAKQIAAGHYFNASCHGTNGTVH \\
\hline & AGPRDTGDDYSPIVKALMSAVEDRGVPTKKDFGCGDPHGVSM \\
\hline & FPNTLHEDQVRSDAAREWLLPNYQRPNLQVLTGQYVGKVLLS \\
\hline & QNGTTPRAVGVEFGTHKGNTHNVYAEHEVLLAAGSAVSPTILE \\
\hline & YSGIGMKSILEPLGIDTVVDLPVGLNLQDQTTATVRSRITSAGAG \\
\hline & QGQAAWFATFNETFGDYSEKAHELLNTKLEQWAEEAVARGGFH \\
\hline & NTTALLIQYENYRDWIVNHNVAYSELFLDTAGVASFDVWDLLPF \\
\hline & TRGYVHILDKDPYLHHFAYDPQYFLNELDLLGQAAATQLARNI \\
\hline & SNSGAMQTYFAGETIPGDNLAYDADLSAWTEYIPYHFRPNYHG \\
\hline & VGTCSMMPKEMGGVVDNAARVYGVQGLRVIDGSIPPTQMSSH \\
\hline & VMTVFYAMALKISDAILEDYASMQSGSGSGSGSGTKLYGDVND \\
\hline & DGKVNSTDAVALKRYVLRSGISINTDNADLNEDGRVNSTDLGIL \\
\hline & KRYILKEIDTLPYKN \\
\hline
\end{tabular}


Table S4. The numbers of expressed enzyme units on the yeast cell surface

\begin{tabular}{lll}
\hline Strain & GA-DocC & GOx-DocT \\
\hline EBY-C $\mathrm{C}_{1}-$ GA-GOx & 38000 & 37000 \\
EBY-C $\mathrm{C}_{1} \mathrm{C}_{1}-$ GA-GOx & 72000 & 37000 \\
EBY-C $_{2} \mathrm{~T}_{1} \mathrm{C}_{1}-\mathrm{GA}-G O x$ & 64000 & 21000 \\
\hline
\end{tabular}

Note: In order to investigate the molecular ratio of GA to GOx on the cell surface, the numbers of expressed enzyme units on the bifunctional yeast cell surface were determined. It was estimated to be about 38000 GA-DocC and 37000 GOx-DocT enzymes per cell (calculated ratio, 1.03:1) when the scaffoldin was CohC-CohT. The correct 1:1 binding ratio demonstrated the proper folding of fusion proteins. As expected, the number of GA-DocC doubled, but that of GOx-DocT retained the same level as CohC domain doubled. However, a downward trend was observed when the scaffoldin was CohC-CohC-CohT-CohC. Specifically, there were about 64000 GA-DocC enzymes and 21000 GOx-DocT enzymes per cell. The calculated ratio (3.05:1) was very close to our designed value (GA:GOx=3:1). 
3. Supplementary Figures:

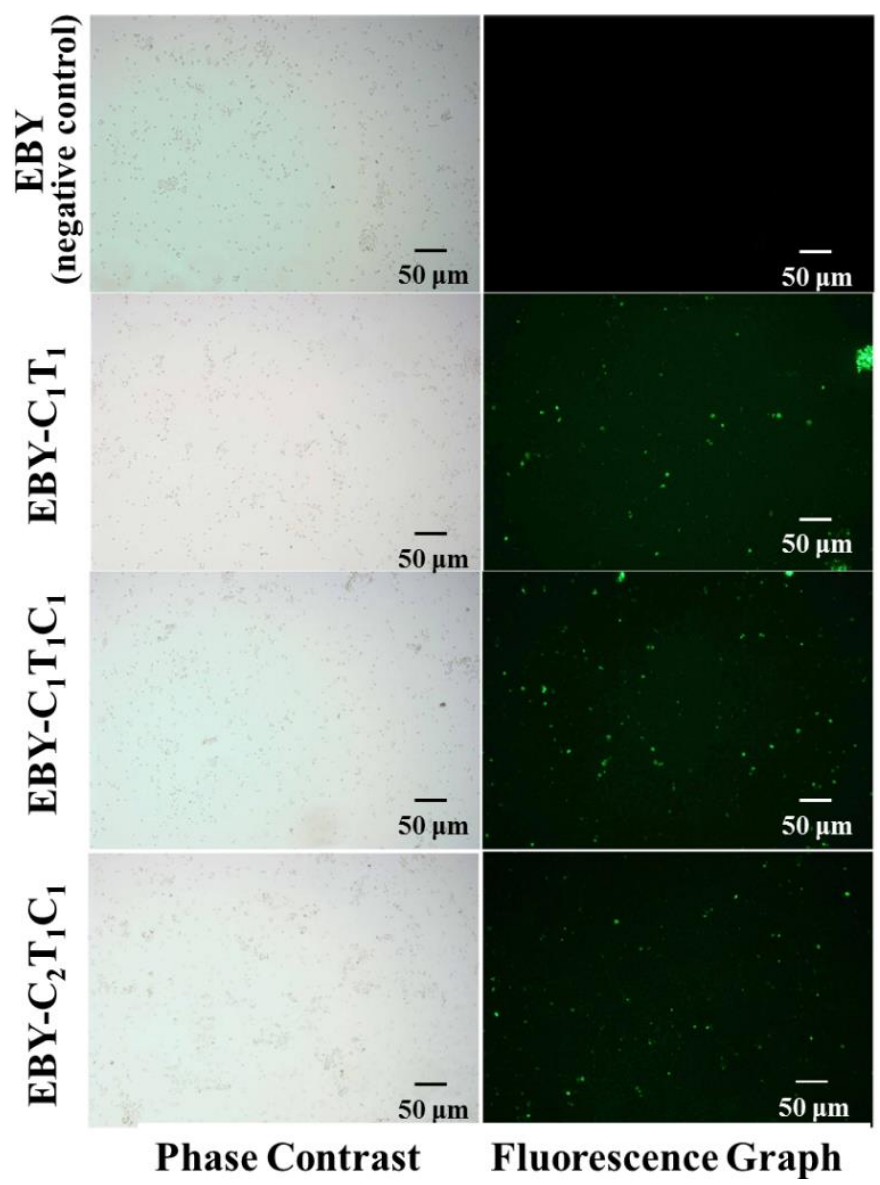

Figure S1. Phase-contrast and immunofluorescence micrographs of yeast cells displaying synthetic scaffoldins 

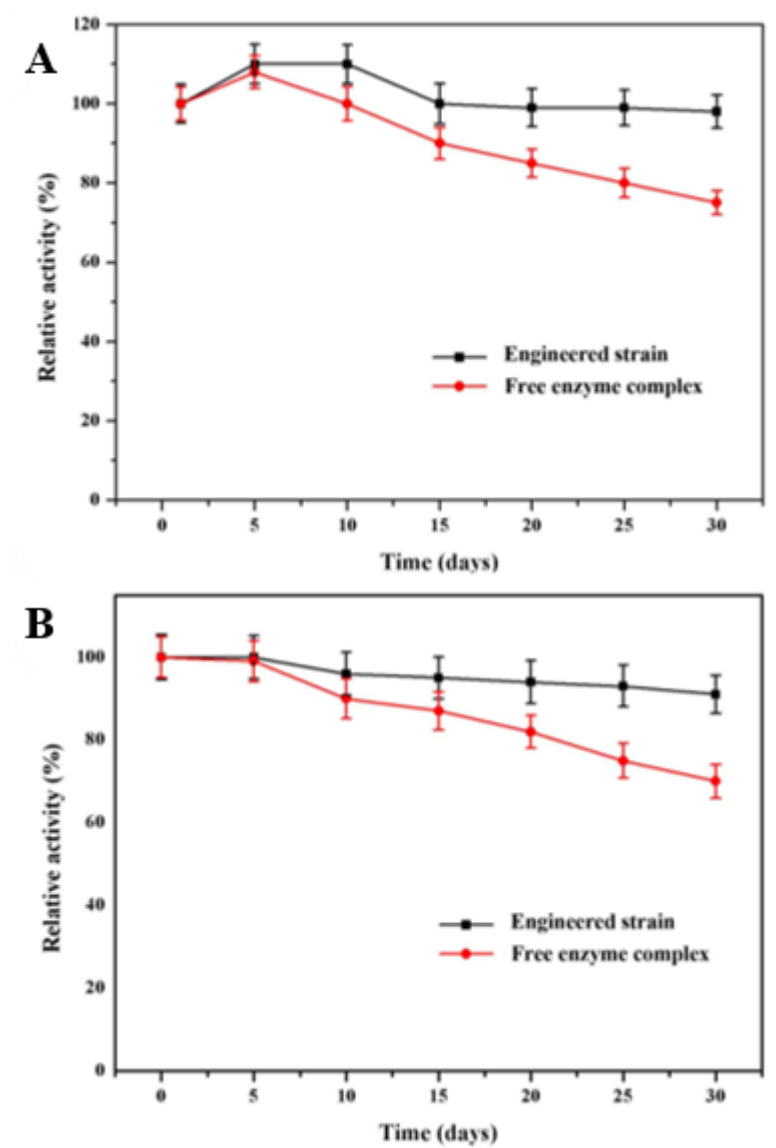

Figure S2. Long-term stability of sequential enzymes co-displayed on cell surface (EBY$\left.\mathrm{C}_{1} \mathrm{~T}_{1} \mathrm{C}_{1}-\mathrm{GA}-\mathrm{GOx}\right)$ and the same amount of free enzyme complex at $4{ }^{\circ} \mathrm{C}(\mathrm{A})$ and room temperature (B). Error bars represent the standard error of three replicates. 


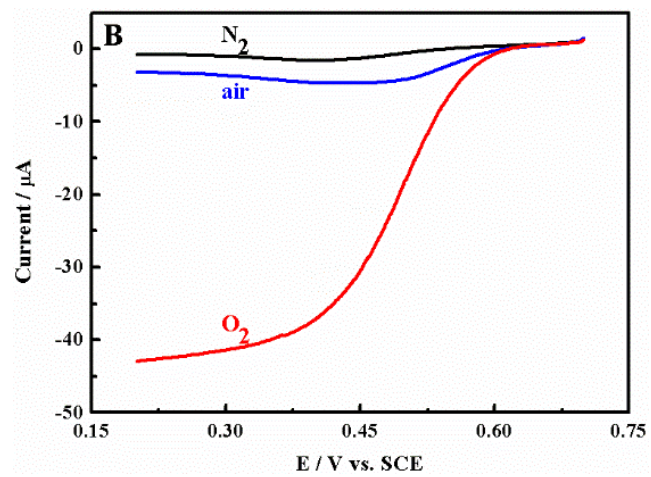

Figure S3. (A) CVs of the Lac/graphene/GCE biocathode in $0.2 \mathrm{M} \mathrm{pH} 5.0 \mathrm{McIlvaine}$ buffer containing $0.5 \mathrm{mM}$ ABTS under (a) $\mathrm{N}_{2}$-saturated atmosphere, (b) ambient air, and (c) $\mathrm{O}_{2}$ saturated atmosphere. Scan rate: $20 \mathrm{mV} \mathrm{s}^{-1}$. (B) Polarization curves of the Lac/graphene/GCE biocathode in $0.2 \mathrm{M} \mathrm{pH} 5.0$ McIlvaine buffer containing $0.5 \mathrm{mM}$ ABTS under (a) $\mathrm{N}_{2}$ saturated atmosphere, (b) ambient air, and (c) $\mathrm{O}_{2}$-saturated atmosphere. Scan rate: $1 \mathrm{mV} \mathrm{s}^{-1}$. 


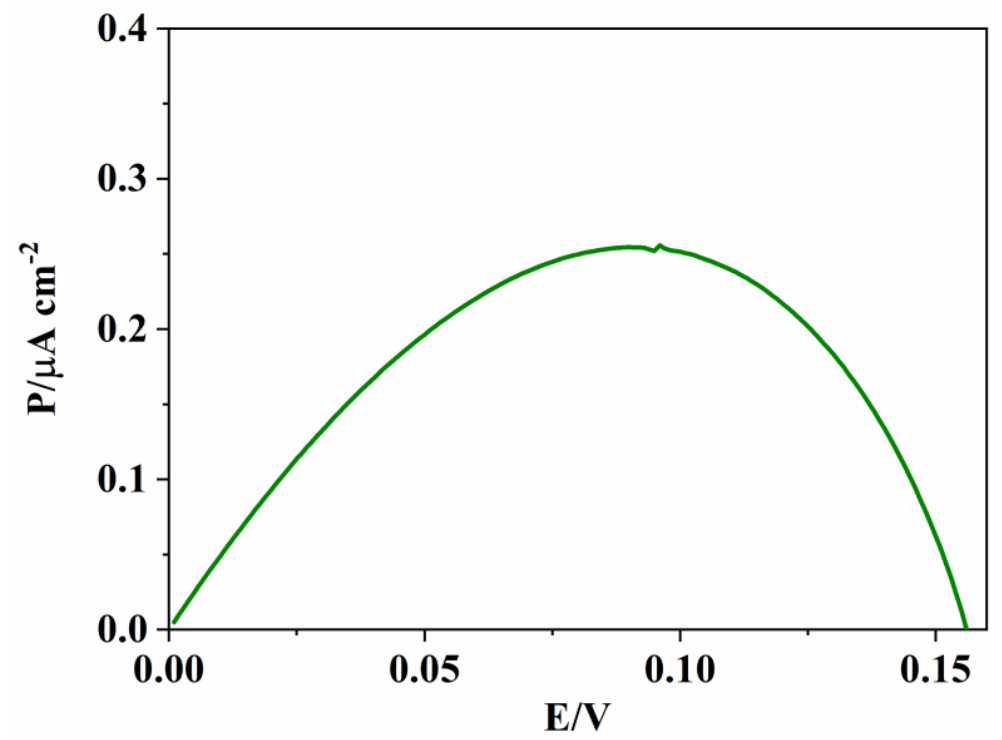

Figure S4. Power density-voltage profiles of the blank control cell consisting of a yeast/graphene/GCE based anode and a Lac/graphene/GCE based cathode. $0.2 \mathrm{M}$ McIlvaine buffer ( $\mathrm{pH}$ 5.0) containing $0.5 \mathrm{mM} \mathrm{MB}$ and $1.0 \%$ (w/w) starch was used for the anode compartment, while $\mathrm{O}_{2}$-saturated 0.2 M Mcllvaine buffer (pH 5.0) with 0.5 mM ABTS was used for the cathode compartment. 

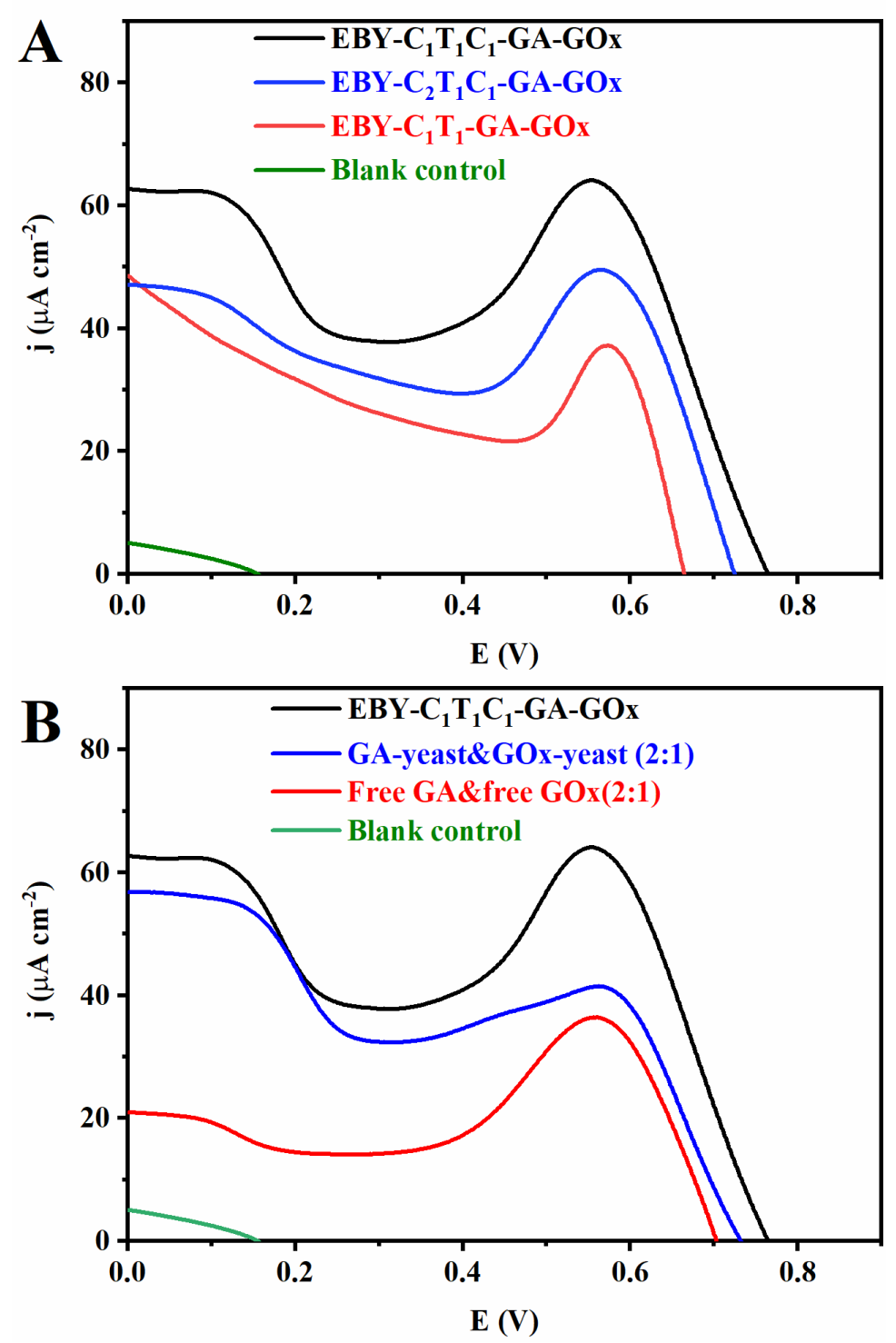

Figure S5. Polarization curves of two-compartment starch $/ \mathrm{O}_{2}$ EBFCs on different bioanodes, which were fabricated by depositing biocatalysts onto graphene/GCE. 0.2 M McIlvaine buffer (pH 5.0) containing $0.5 \mathrm{mM} \mathrm{MB}$ and $1.0 \%(\mathrm{w} / \mathrm{w})$ starch was used for the bioanode compartment, while the Lac/graphene/GCE was used throughout as the biocathode, in which $\mathrm{O}_{2}$-saturated 0.2 M Mcllvaine buffer ( $\mathrm{pH}$ 5.0) with 0.5 mM ABTS was used for the biocathode compartment. Blank control indicates a cell consisting of a yeast/graphene/GCE based anode and a Lac/graphene/GCE based cathode. 


\section{References:}

[1] H.W. Wang, Q.L. Lang, L. Li, B. Liang, X.J. Tang, L.R. Kong, M. Mascini, A.H. Liu, Yeast surface displaying glucose oxidase as whole-cell biocatalyst: construction, characterization, and its electrochemical glucose sensing application, Anal. Chem., 85 (2013) 6107-6112.

[2] A.S. Wieczorek, V.J.J. Martin, Effects of synthetic cohesin-containing scaffold protein architecture on binding dockerin-enzyme fusions on the surface of Lactococcus lactis, Microb. Cell Fact., 11 (2012) 160.

[3] S.H. Lee, J.H. Choi, S.H. Park, J.-i. Choi, S.Y. Lee, Enantioselective resolution of racemic compounds by cell surface displayed lipase, Enzyme Microb. Technol., 35 (2004) 429-436. 